\title{
Communications, Multimedia, Ontology, Photonics and Internet Engineering 2012
}

\author{
Ryszard S. Romaniuk
}

\begin{abstract}
Wilga Symposium gathers two times a year, together around 300 young scientists active in advanced photonics, electronics and software systems, including Internet engineering. May 2012 marked a jubilee XXXth meeting in Wilga Resort. There were presented over 250 papers from nearly all technical universities in this country. The organizers present here a research survey of the WILGA Symposium work. Also a digest of chosen Wilga references is presented [1]-[39]. This paper is the second part of a concise review digest focused on optoelectronics, communications, multimedia and ontology of information technologies.
\end{abstract}

Keywords-Photonics, optical fibers, optoelectronics, communications, multimedia, cryptography, virtual worlds, ontology of information systems.

\section{INTRODUCTION}

$\mathbf{T}$ HE XXX ${ }^{\mathrm{TH}}$ JUBILEE SYMPOSIUM of young scientists WILGA 2012 on Photonics and Internet Engineering has gathered together over 300 participants in January and May Editions. There were presented over 250 papers - mainly concerning the realized Ph.D. theses and participation in research projects relevant to the topical area of the meeting. There were also presented a few plenary papers introducing the audience into new research areas of photonics and electronics. The symposium is organized under the auspices of SPIE - The International Society for Optical Engineering, IEEE Poland Section, Photonics Society of Poland, KEiT PAN, PKOpto SEP and WEiTI PW. The symposium is organized annually by young researchers from the PERG/ELHEP Laboratory of ISE PW with cooperation of SPIE and IEEE Student Branches.

\section{TOPICAL TRACKS OF WILGA 2012}

The topical session and tracks of WILGA 2012 were as follows: nanotechnologies and nanomaterials for optoelectronics and photonics, optical fibers for sensors and all-photonic devices for sensors, active optical fibers, sensors and sensory networks, object oriented design of optoelectronic and photonic hardware, photonics applications, advanced bioelectronics and bioinformatics, co-design of hybrid photonic mechatronics and electronic systems, computational intelligence in optoelectronics and robotics, development in the wide-angle astronomic observations of the whole sky - pi-ofthe-sky project, processing and imaging of multimedia data

Ryszard S. Romaniuk is with Warsaw University of Technology, Institute of Electronic Systems, Nowowiejska 15/19, Warsaw, Poland (e-mail: rrom@ise.pw.edu.pl). streams, machine vision, vehicles - quadrocopter and Mars rover, analog transmission systems in noisy conditions with digital reverse transmission channel, optoelectronic and photonic metrology, reconfigurable measurement systems, high performance - low-jitter low-latency transmission systems - White Rabbit, thermonuclear fusion experiments - JET and ITER, research results update from HEP experiments TOTEM and CMS/LHC in CERN, integration of software and hardware.

\section{Liquid CRYSTAL OpticAl Fibers}

Classical Liquid Crystal Optical Fibers: Liquid crystal optical fibers (where a core is a liquid crystal) are investigated for functional photonic components compatible with standard SM (single mode) optical fiber transmission systems. The values of elastic constants in nematic liquid crystals play a role in certain solutions of sensors and modulators as well as nonlinear photonics solutions. The research on liquid crystal optical fibers and nonlinear photonics is intensely carried out at Faculty of Physics WUT by teams of prof. T. Woliński, prof. A. Domański and prof. M. Karpierz and dr A. Rutkowska. The liquid crystals (LC) used in optical fibers and nonlinear photonics are: smectic (SLC), nematic (NLC), and chiral nematic (NLC*). Reorientation of the modules, in reference to initial orientation, is influenced by an external electric field. Initial configuration in NLC cells can be essentially homeotropic and planar. In optical fiber the orientation against the long fiber axis can be: planar, axial, radial and transverse, as well as mixed. Fundamental deformations of NLC, visible over certain threshold value of the excitation field, are: splay, twist and bend, with the involvement of three elastic constants $k_{11}$, $k_{22}$, and $k_{33} . k_{11}$ is responsible for the Frederics effect. Measurement of these constants base on electro-optical effect, but also nonlinear self-diffraction method, z-scan and all optical method. For self-diffraction method and homeotropic texture $\left(k_{33}\right)$ the polarization is not important. For self-diffraction method and planar texture $\left(k_{22}\right)$ polarization is perpendicular to initial orientation of long axis of molecules. Self-diffraction method of measurement of Frank elastic constants is as good as traditional method with external electric field. LC display electrical, magnetic, optical and mechanical anisotropies. After some threshold temperature the anisotropies disappear. In optical domain the ordinary and extraordinary refractions coalesce abruptly to a single isotropic refraction. Mechanism of optical nonlinearity in LC stems from reorientation, thermal and Kerr nonlinearities. Time responses to these phenomena in the LCs are of the order of ms. 
Photonic Crystal Fibers impregnated with LC: Photonic crystal fibers (POF), or holey optical fibers, can be impregnated or infiltrated with liquid crystals (LC), leading to a new class of Photonic Liquid Crystal Fibers (PLCF). This is an interesting and alternative way of making highly nonlinear optical fibers, to the fibers of classical core cladding construction, where the core is filled totally with a liquid crystal. The POF have micro/nano capillaries not filled, as usual, by air but by liquid crystal. Filling a POF with LC changes dramatically it propagation characteristics (refractive, dispersion, spectral attenuation and nonlinear). Potential applications of PLCF are: all optical switching, light beam control and steering, amplification, nonlinear directional couplers, tunable filters, phase shifters, frequency conversion, etc. Classical POF can be, depending on construction - dense or rare, highly nonlinear, highly dispersive, endlessly single-mode, polarization maintaining, high or low numerical aperture, etc. There are two light propagation mechanisms in a POF, also depending on the fiber construction: mTIR - modified total internal reflection, and PBG - photonic bandgap. mTIR POF are broadband, while PBG POF are narrowband for optical wavelengths. The measurements of PLCF (dr K. Rutkowska, Physics, WUT) indicate that: power of light beam affects the nonlinear behavior of fibers; fibers can be considered as a matrix of waveguide channels; spatial light localization is observed, when a discrete soliton is formed. These results may lead to practical photonic devices.

Nematicons - spatial solitons in nematic liquid crystals: Nematicons are spatial solitons in nematic liquid crystals. Due to large value of nonlinear index $n_{2}$ the power of soliton for NIR in liquid crystal is on the level of $\mathrm{mW}$, instead of $\mathrm{kW}$ in GaAlAs, MW in $\mathrm{SiO}_{2}$ and $\mathrm{kW}$ in polymer fibers. Isotropic phase of LC has random orientation of molecules while nematic is strictly oriented in parallel. Birefringence of typical nematics is 0.2 , while in special mixtures this increases to 0.7. Birefringence is defined as a difference between extraordinary and ordinary refractive indices. Basic orientations of LC molecules against the optical fiber axis are: parallel, axial, transverse and perpendicular, or in other words - textures: homeotropic, planar, planar+v, hybrid and twist/chiral. Self focusing effect stems from making a gradient waveguide by the beam itself. Chiral nematics create effectively layers (a pitch) with the thickness equal to the period of the molecule twist. Chiral structures are equivalent to periodic discrete diffraction elements with added nonlinearity (nonlinear waveguide arrays). In such structures the nematicons can interact mutually, change the input beam polarization and position, be influenced by the external field, be rerouted (switched) between waveguide branches, etc. There are some differences between high and low birefringence NLCs in these characteristics. Nematicons in chiral nematics (M. Karpierz): do not need external fields, require $\mathrm{nW}$ of light power, creation time is of the order of ms, can guide probe beams, can be redirected by changing light power - by other nematicon, by external electric field, by changing beam polarization, the creation mechanism can be discrete or classical diffraction in multiple layers, can be optimized by changing the pitch and birefringence.

\section{Medical Applications of Active Optical Fibers With Photosensitive DRUGS}

Optical fiber technology is developed at Białystok University of Technology, Faculty of Electrical Engineering, in Optoelectronics Laboratory chaired by prof. J. Dorosz and prof. D. Dorosz. One of the projects concerns upconversion emission in double-clad tellurite optical fibres. The purpose is to enrich medical and bioscience applications of optical fiber lasers. Such optical sources are applied for optical response embracing therapy, biostimulation, cosmetics and diagnostics, as well as thermal response, including surgery and aesthetic medicine. The applications include: photodynamic therapy, hair and tattoo treatment, photochemical diagnostics, endoscopy, OCT imaging, spectroscopic diagnostics, surgery in urology, abdominal, dental,..., skin resurfacing, birthmarks, dermatology, etc. Biostimulation is a fast growing field os applications of fiber lasers. Biostimulation mechanism is as follows: photon, mitochondrium, increase ATP production, activity increase $\mathrm{T}$ lymphocyte, fibroblast, heparin unbinding, tissue energy increase, vitality increase. Biostimulation is used not only in dermatology but also endoscopically in gastroenterology, pulmonology, gynaecology, and in many other medical fields. The used light sources are He-Ne $(633 \mathrm{~nm})$, InGaAlP (635nm, 650nm, 660nm, 670nm) GaAlAs (780nm, $820 \mathrm{~nm}, 830 \mathrm{~nm})$, GaAs $(904 \mathrm{~nm})$. Photodynamic therapy bases on application of a photosensitive drug and optical activation of this drug. Activated drug releases locally radicals. The process leads to very localized cell necrosis. Various photosensitive drugs have different activating wavelengths, usually spanning from $630 \mathrm{~nm}$ to $690 \mathrm{~nm}$. Some of photosensitive drugs are: natrium porfimer, BPD-MA, m-THPC, 5-ALA, HPPH, boron-photoporfirines, lutetium teksapiryne, ftalocyjaninum4, natrium taporfinum, and others. Fiber lasers are used in optogenetics. Nerve fibers are stimulated electrically and then undergo optogenetic excitation followed by optogenetic inhibition. Specialized, medical oriented optical fiber equipment is constructed for these purposes.

Thulium and Ytterbium doped double clad telluride active optical fibers: Double clad optical fiber radiating in visible spectrum is subject of research for medical applications in Optoelectronics Laboratory at Białystok University of Technology (prof. D. Dorosz). Tellurite glass of low phonon energy, equal to $750 \mathrm{~cm}^{-1}$, is used as a matrix. Thulium is used as an acceptor, for the up-convesion quantum transition present in the visible spectrum. Ytterbium is used as a sensitizer for increase in the luminescence band intensity. The following tellurite glass is subject to synthesis: $\mathrm{TeO}_{2}-\mathrm{GeO} 2-\mathrm{PbO}-$ $\mathrm{BaO}-\mathrm{Nb}_{2} \mathrm{O}_{5}-\mathrm{laF}_{3}-a \mathrm{Yb}_{2} \mathrm{O}_{3}-b \mathrm{Tm}_{2} \mathrm{O}_{3}$, where $a=1 \%$ mol, $b=0.025--0.2 \%$ mol. The glass is melted in $900^{\circ} \mathrm{C}$ in protective inertial Ar atmosphere. Then it is annealed in $350^{\circ} \mathrm{C}$. The obtained glass parameters are: mass density $6.21 \mathrm{~g} / \mathrm{cm}^{3}$, refractive index 2.074 , spectral transmission 0.35 $5.5 \mu \mathrm{m}$, thermal expansion coefficient $108.9 \times 10^{-7} \mathrm{~K}^{-1}(100-$ $400^{\circ}$ ), transformation temperature $T_{g}=345^{\circ} \mathrm{C}$, Dilatometric softening point $T_{s}=364^{\circ} \mathrm{C}$. Optical, mechanical and thermal properties of the glass were measured as functions of $\mathrm{Yb}^{3+}$ and $\mathrm{Tm}^{3+}$ ions concentrations. The major absorption peak is 
for around 990nm for a glass with $1 \% \mathrm{~mol} \mathrm{Yb}^{3+}$ and $0.25 \%$ mol $\mathrm{Tm}^{3+}$. Secondary peaks are for around $700 \mathrm{~nm}, 800 \mathrm{~nm}$ and $1220 \mathrm{~nm}$. The major luminescence peak is for $474 \mathrm{~nm}$ and $650 \mathrm{~nm}$. A double clad fiber was manufactured from active telluride glass. The parameters are: $\mathrm{NA}_{r}=0.32, \mathrm{NA}_{p}=0.95$, active core diameter $70 \mu \mathrm{m}$. Fiber diameter $320 \mu \mathrm{m}$. The fiber has emission in blue.

\section{Optical MEASUREMENTS}

Reference tests of cameras with calibrated light source: A measurement system is under construction with feedback controlled light source and photometric integrating sphere for reference tests of cameras for astronomical applications. Light source radiation shifts towards shorter wavelengths and the intensity increases with the increase in $T$. The feedback relies on measurement the Planck curve (for a few wavelengths) of the source and determining its temperature. The measurement system consists of Ulbricht sphere, photodiodes, spectrometer, shutters, halogen light source. Shutters are coarse and fine, driven by stepper motors for intensity regulation. The driver is based on ATmega64 processor. The light source work conditions are set basing on the measured spectrograms. Cameras are scheduled to be tested with the calibrated light source.

Optical Diffraction Tomography: Optical tomography creates a digital volumetric model of an object by reconstructing images made from light transmitted and scattered through the object. Thus it is applied to at least partially light transmitting or translucent objects, like for some kinds of medical imaging (OCT). There a lot of variants of optical tomography (time of flight, i.e. time resolved, coherence, spectral, etc). In optical diffraction tomography incident optical field shines on a rotated object, is transmitted through it distorted, then is focused by imaging lens on a CCD camera. Object rotation introduces a runout error due to out of focus effect. The runout error can be corrected by numerical refocusing of the image, a research work which is done in group of prof. M. Kujawinska (Mechatronics, WUT). Runout error limits tomographic measurements of microstructures. Minimization of runout effects with numerical correction algorithm by: reduction of diffraction and geometrical distortions of reconstructed structures, reduction of unwrapping errors. ODT with corrected runout error allows for reconstruction of micrometer scale structures.

\section{Photonic and Electronic Sensors, Components AND CIRCUITS}

Organic short wavelength lasers: Short wavelength lasers (SWL) are used for optical data storage, reprographics, biotechnology, color displays, plastic optical fibers, remote sensing of chemical agents. They feature high brightness and improved color saturation. SWL are manufactured as wide bandgap diode lasers (nitrides), by frequency mixing (SHG - second harmonic generation and SFG sum frequency generation) or using optical up-conversion. There are also possible FHG - fourth harmonic generation and DFG difference frequency generation effects. The optical set-up with SHG, SFG etc. is sensitive to alignment. The optical fiber technological teams from UMCS Lublin and IMiO PW are working on PMMA doped with active nano-powder. The PMMA is used for some time as an efficient optical material. It has high energy phonons among the polymers. Here it is used as a matrix. It is transparent and thermoplastic, low cost, is easy to manufacture. It possesses reasonable attenuation, forms well developed polymer waveguides, is flexible and has high mechanical strength. The used nanopowder is $\mathrm{Pr}^{3+}$.It has transitions in the blue, green and red wavelengths. It exhibits up-conversion in the visible in combination with $\mathrm{YF}_{3}$. The PMMA doped with nano-powder was researched for its structure and was optically characterized. There were made comparisons of luminescence properties between several different composites $\mathrm{Pr}^{3+}: \mathrm{LaAlO}_{3}$ and $\mathrm{Pr}^{3+}: \mathrm{YF}_{3}$. Excitation and emission spectra were measured. The results reveal that the PMMA was homogenously doped and that the nanopowder crystallites shield the active ion $\mathrm{Pr}^{3+}$ from external PMMA influences. The crystallites do not influence the optical transitions significantly. Thus, the doped PMMA seems to be a promising material for not too long future light sources and amplifiers.

Photonic bio-fuel inspection: Photonic, spectroscopic biodiesel sensors are subject of research by the research group in IMIO WUT (dr M. Borecki). The initial design idea stems from the following statement. There are many standards of fuel examination, but they are made exclusively for fuel producers. At present, one of the most important bio-fuel types due to ecological demand, is the bio-diesel fuel. The aim of research is to build cheap, mobile and efficient, photonic bio-fuel quality sensor basing on optical spectroscopy. Cost of the calibrated light source contributes most to the overall value of the systems available on the market. The assumed solution aims at the modularity of the source. The fuels under analysis contain $\mathrm{C}, \mathrm{H}, \mathrm{H}_{2} \mathrm{O}, \mathrm{N}_{2}, \mathrm{~S}, \mathrm{Cl}, \mathrm{O}_{2}, \mathrm{SiO}_{2}$ and their compounds. The diesel fuel contains 20 major components and about 200 minor components that may be detectable by the photonic sensing system. Fuels are categorized against: density, calorific value, origin, $\mathrm{H} / \mathrm{C}$ ratio, etc. The following parameters are determined for diesel fuels: RVP - Reid vapor pressure, boiling point, flash point, density, number of carbon atoms in particle and viscosity. The intelligent portable multifuel (gasoline and bio-fuel), multi-sensor, photonic-electronic analyzers base mainly on computerized processing of the near UV, optical and IR spectra, and applying mathematical models for determination of cetane number and as many as other 40 parameters. A simple and cheap sensor is also needed measuring very fast only a few key parameters and determining the fuel usability. The novel design bases on optoelectronic interface working at non-typical frequency eliminating the background radiation, fiber optic pigtails, and capillary optrode used for photo-spectrometric analysis of the attenuation and fluorescence. Characteristic features of the spectrum to be analyzed for fast bio-fuel quality results are:

- attenuation features: $260 \mathrm{~nm}$ features indicating the concentration of bio-components, 480nm - presence of lubricants, $620 \mathrm{~nm}$ - presence of unsaturated fatty acids;

- fluorescence excitation for 260nm: 270-300nm features - showing concentration of bio-components; 
- fluorescence excitation for $365 \mathrm{~nm}$ : $400 \mathrm{~nm}$ - presence of washing agent, $480 \mathrm{~nm}$ - concentration of $\mathrm{Cl}$ based improvers.

The design of a cheap photonic bio-fuel analyzer bases on building light sources and re-connectable optoelectronic units that are able to separate efficiently all these above listed analog signals, digitize them immediately after detection and process for the useful results. The optoelectronic set-up of the fuel quality sensor consists of: function generator, light source, optical coupling, detectors, digital electronics like DaqLab, PC with DaisyLab or similar software, Ethernet access.

Wireless/photonic sensor networks: Research is continued (ISE WUT) on wireless telemetry systems based on mesh networks which are compatible with the IEEE 802.15.5 standard (commonly referred to as ZigBee). A number of remote nodes is communication wireless over $2.4 \mathrm{GHz}$ bandwidth or optically to a concentrator connected in turn via the RS, USB, Modbus etc to a PC. The nodes feature a rich collection of the IOs digital and/or analog. Slow control of the system can be done via the RS.

Photonic inspection of yeast: Optical fiber capillaries were applied for measurements of spectrophotometric properties of Saccharomyces Boulardi SB. SB is a species of tropical yeast isolated from the peel of lychee and mangosteen fruits. SB is a probiotic, unicellular and globular. It reproduces by budding and grows at very high temperatures of $37^{\circ} \mathrm{C}$. The interest in $\mathrm{SB}$ is because it is the easiest available type of yeast. However, it causes inflammation and some disorders in human body. The results of human cytology are obtained after two weeks. SB shows high level luminescence in the short-range UV $375 \mathrm{~nm}$. The laboratory set-up consists of a PC controlled spectrophotometer, optical fiber capillary with a sample, and light source with a power supply. The yeast samples were measured with and without annealing. The aim of the design is to build an automated, cheap sensor for fast SB yeast detection.

Photonic lab-on-a-chip bio-chemical sensors: Lab-on-a-chip principle is: multiple functions of a chemical lab are put together in single chip; operations with extremely small volumes of reagents due to high sensitivity and use of microfluidic circuits, a variety of types of used sensors - electronic, MEMS, optical fluorescence, photonic, and plasmonic. Operation principle can be refractometric by measurement of effective optical index change due to monitored bio-chemical reaction but also other like nephelometric. The configuration are: MZI - Mach-Zender, FP - Fabry Perot cavity, DPI - dual polarization interferometer. A full featured photonic integrated sensor system was presented by dr A. Kaźmierczak of TNO Delft, performed inside the European FP6-IST Sabio project. The system consists of: optical interrogation device, couplers, multichannel sensor topology, simultaneous interrogation of numerous sensor channels, on-chip signal splitting by miniature vertical grating coupler, off chip solutions, and multichannel detector. The results of measurements indicate high tolerance and coupling repeatability of optical coupling during the bio-chip replacement. The following tests were done: refractometry measurements for anti-BSA protein, temperature influence compensation, etc. A complete optical solution for photonic bio-chemical sensing system has been proposed including transducer design, optical integrated multichannel circuit and the integration platform.

Intelligent Pipeline ADC: Pipeline ADC have interesting characteristics indicating their applications in many applications. Adaptive estimation algorithms can be used to improve ADC performance, including the increase in the ENOB, while not or minimally increasing their complexity. Implementation of the approach is possible in a new class of ADC, so called intelligent adaptive cyclic/pipeline ADC (IPADC) in which output codes are computed as binary words of the fixed length (dr K. Jędrzejewski). Optimization of IPADC relies on finding, for every converter stage, the maximal value of gain, and corresponding value of digital coefficient, which minimize the mean square error of the estimate. The parameters have to satisfy the statistical fitting condition, guaranteeing that the probability of saturation does not exceed an assumed (and/or given) sufficiently low level. In classical pipeline ADC, the problem of overloading is solved by artificial decrease of the gain in an individual stage and resignation from erroneous bits in sub-codes. In IPADC the gain may assume any value, not only integer power of two. Greater gain and optimal conversion algorithm allows to achieve the final ENOB value in IPADC greater than in standard pipeline ADC. The disadvantages of IPADC are: high requirements on resolution and accuracy of the internal DAC. The gain is increasing in subsequent stages of the IPADC. A modified algorithm for IPADC was implemented which omits these disadvantages. For each stage of conversion there are performed estimates of input sample and maximal coefficients of the amplifier gain are calculated. In modified IPADC the resolution of the DAC sub-converter is low and equal to the resolution of the ADC sub-converter. Gain of the amplifier is smaller than in standard IPADC. As a result the following was obtained: simplification of the IPADC architecture, lowering size, power, costs, and increase in ENOB. ENOB is limited only by the level of internal noise (errors). Simulation tools enable assessment of a number of ADC stages, a number of bits in digital parts and other parameters required to obtain an assumed ENOB value.

Inkjet printing of electronic and photonic circuits on LTCC: Inkjet printing is increasingly frequently used as a technology of choice for photonic and electronic components, sensors and circuits. A team lead by prof. M. Jakubowska of WUT and ITME is working on methods of making electric connections using inkjet printing on LTCC (low-temperature cofired ceramic) substrates. LTCC substrates are commonly used for multilayer electronic PCBs by the electronic industry for aerospace, automotive, medical and telecommunication applications. Multilayer hybrid ceramic circuits consist up to 100 layers and contain for example mounting holes, embedded active elements, printed embedded passive components like resistors, capacitors, coils, conductive tracks, VIA holes etc. The fabrication process includes the following steps: green tape foil, cutting, hole drilling, VIA filling, printing, stacking, lamination, prefiring, firing, tests, finished module, packaging. Screen printing is widely used in LTCC technology. Using screen printing in LTCC circuit manufacturing brings several disadvantages: difficulties in printing on soft, porous, fragile, unfired LTCC foil; the line width limit is $0.1 \mathrm{~mm}$ ' not enough 
alignment precision; VIA diameter limit is $0.25 \mathrm{~mm}$ ' expensive tools are needed, difficulties in mounting fragile LTCC foil in screen printer. The aim of the research is to develop contactless method of fabrication electrical connections in hybrid electronic circuits on LTCC substrates using inkjet printing technique. Proposed solution is expected to aid essentially electronic industry with hybrid electronic and photonic devices of new generation. Circuit scale integration will increase along with decrease of fabrication costs. This solution is expected to enable rapid prototyping of hybrid electronic and photonic modules. The advantages of this technology is: contactless, printed lines width lower than $0.1 \mathrm{~mm}$, alignment precision better than $5 \mu \mathrm{m}$, effective VIA filling, 3D surface printing enabled, additive and waste free process, low power consumption, no additional tools needed and low cost rapid prototyping. Polygraphic inkjet printers and inks have to be modified to be usable for electronics industry. The effective printer has to be able to align printout with precision better than $2 \mu \mathrm{m}$, work with commercially available print heads, use organic and inorganic inks in a wide range of $\mathrm{pH}(1-11)$ and viscosity 1-12 cPas values, generate single drops of volume in the range of 35-150pl, pause and stop at any time. The investigated parameters are roughness and porosity and their influence on resistance, track/drop shape and mechanical endurance.

Reference atlas of Copper standard spectrum: The team from Rzeszów University of Technology (dr J. Domin, Rzeszów Univ. Technology) specializes in making precise reference spectra for molecular research. 1300 lines/mm Eschelette type grating is used mounted at PGS-2 spectrograph. WU1 plates by Wephota were used for expositions. The goals are: elaboration of spectral standard in Schumann region; measuring of high precision atomic standard lines fore this spectral region for source catalogues. Using copper atomic spectrum for standardization of the investigated spectra it is hoped to receive a precision of a range better than $0.003 \AA$. It is standard precision which is required in molecular spectroscopy. Having such precision one can hope for possibility of calculations of molecular parameters.

\section{COMmunicATIONS}

A topical track on photonic Internet consisted of papers on photonic LANs, trunk optical lines of ultimate throughput, new optical non-blocking codes, developments in all-optical architectures. One of the concepts, not yet fully confirmed, is application of organic photonics to build the framework of the variety of access networks to the terabit photonic transport network. Micro-structured plastic optical fibers - mPOF are under intense studies, also in this country. They are cheap and are user scalable, even in singlemode version.

To meet new demands, the next generation of highperformance wireless and optical networks and communication systems (CS) must support a significant increase in data rates, better coverage, greater spectral efficiency, and higher reliability. The applications of high performance data transmission networks extend well beyond traditional understanding of telecom systems. Complex networked structures are fueling the development of self configurable sensory meshes, smart structure, bioengineering including body area networks, etc. These structures using wireless and optical technologies need extreme energy efficiency and introduce completely new wireless networking paradigm. Overcoming these technical challenges will require significant breakthroughs in wireless and photonic/optical component and system design. A dominating tendency in all these domains is to go digital, independently of the fields of applications, including short distance extremely distributed communications. The reason is obvious. The known and traditional analogue solutions are much less efficient and noise resistant than digital systems, have low bit rate and non-optimally utilize the available channel bandwidth. Theoretically, however, there is a wide class of practically important and potentially of extensive applications analog CS. These systems can potentially beat digital ones for similar applications. Principal differences between analog and digital CS are as follows. Transmitters of digital CS contain digitizing and coding units, which cannot be described by a continuous input-output functions. This does not allow to determine explicit form of the probability density function, which describes the signal transmission in the CS in the presence of noises. Transmitters of analog CS contain analogue modulators, and in the most advanced systems only they also contain sample and hold units. All the systems consisting of transmitter with S\&H units, channel and receiver can be described by continuous input-output functions and allow for definition the explicit form of the probability density function, which describes the signal transmission in the CS in the presence of noise. This is a profound difference between the analogue and digital CS (ACS and DCS). It results in different measures of the transmission quality. For DCS, the basic analytical measure of the quality of transmission is men square error of the output estimates of the input signal. This measure cannot be simply formulated. For ACS, the mean square error of output estimates can be formulated analytically, because the probability density is determined. This difference has divided ICT into two directions. The DCS has concentrated on development of the increasingly efficient methods of discretization, coding, keying and reversed operations in receivers. The ACS remained a niche. There is no general criterion of transmission quality in DCS. Three separate non-general criterions, related by internal parameters, are used instead for determination of DCS quality. These are: power efficiency of transmission measured in $\mathrm{J} / \mathrm{bit}$, channel bandwidth efficiency measured in $\mathrm{bit} / \mathrm{s} / \mathrm{Hz}$, and bit error rate (called commonly as the P-B efficiency). The closeness of these two first parameters is estimated to the Shannon's boundary. BER is assessed additionally using known characteristics of the channel noise, its bandwidth, gain and power of the transmitter. The aim of optimization of DCS is maximize bandwidth efficiency $\mathrm{B}_{e}$ in bit/s/Hz, minimize energy (power) efficiency $P_{e}$ in $\mathrm{J} / \mathrm{bit}$, minimize BER expressed in probability of erroneous transmission per a single bit. Optimal DCS should transmit signals for the maximal possible distance with maximal rate, quality, and reliability and should have minimal complexity, cost and energy consumption. Shannon theorem for Gaussian channels says that there exist encoding schemes permitting to transmit the signals at the power - bandwidth efficiency 


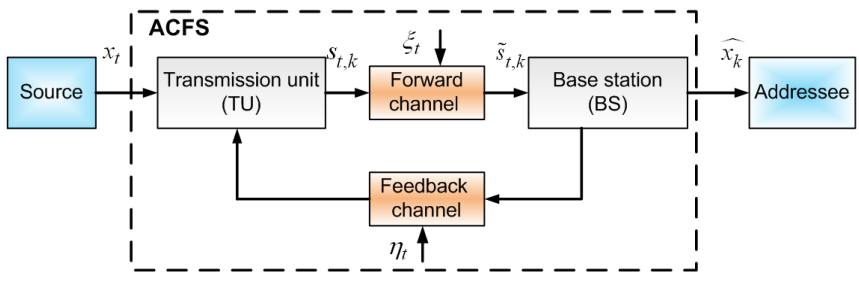

Fig. 1. A basic simplified block diagram of the AFCS. $x_{t}$-input signal, $x_{k}$ estimates of the input signal, $\eta$-noise in the forward and feedback channels, s-signals transmitted and received in the forward channel. Forward and Feedback channels may be RF, open optical or cable - fiber optic.

attaining Shannon boundary with the infinitesimally small frequency of errors. Simultaneously it says that transmission at a greater efficiency is impossible. The characteristics of optimal DCS are: signal transmission is with bit rate equal to the capacity of the channel; power-bandwidth efficiency is at the Shannon boundary; they fully use their power/bandwidth resources for a given BER; no analytical description method exists for DCS. In practice the best DCS solutions have their parameters of 3-5dB far away from the Shannon's boundary. Adaptive feedback communication systems AFCS: Adaptive feedback communication systems, especially in fiber optic version, are of interest for ultimately high quality mixed analog - digital systems (prof. A. Platonov of ISE WEiTI PW), Fig. 1. Data transmission performance in AFCS depends on characteristics and parameters of: source, forward and feedback channel, transmission unit (TU) and adaptive modulator (AM), received signals processing algorithm. Source parameters are: mean value of the input signal, variance (mean power) of the input signal, band frequency of the input signal. TU and AM modulator parameters are: amplitude of a signal transmitted by $\mathrm{TU}$ into a channel, saturation parameter of $\mathrm{TU}$, number of samples being sent, variance of the errors in the feedback transmission. Parameters of the channels are: bandwidth of the forward channel, variance of the noise in the forward channel, distance between TU and BS, channel gain. Parameters of the signal processing algorithm in the BS are: amplitude of the signal on the BS input, the maximum number of transmission cycles for each sample, variables of BS algorithm. Building a testing workbench for such defined AFCS allows to study novel effects in optimal AFCS. It is used for analysis of quality and efficiency of transmission depending on conditions and fields of optimal AFCS applications. Expansion of the analysis tool is for multimode AFCS transmission systems (AFCS phonic networks).

Current issue of AFCS is their optimization. It is assumed that each sample of the input signal is transmitted iteratively, in $\mathrm{n}$ cycles, in the same way and independently from the previous samples. Analog AFCS has an adaptive AM modulator with nonlinear characteristics. Analytical form of general criterion of transmission quality (MSE) is possible using simple mathematical models of the signal source, adaptive modulator, forward and feedback channels and receiver in base station (BS). Thus, an algorithm exists of transmission and reception, where there are embedded all system parameters and controls, which minimizes the MSE of estimates of the signal for each cycle of the sample transmission. The algorithm obviously bases on adaptive adjusting of the TU and signal processing in the BS, starting the process from assumed initial conditions. The expected result of optimal analog AFCS, treated as a generalized communication channel, is that the system works precisely on the Shannon's boundary for each cycle of adaptive and recurrent signal transmission. Such result is unachievable in digital CS. However, expansion of the signal spectrum greater than the threshold coefficient decreases the P-B efficiency of transmission. Optimal analog AFCS realize theoretically lossless information transmission. While, digital AFCS transmission is always combined with partial loss of information due to quantization of transmitted signal. High quality ADCs asymptotically improve the digital AFCS parameters to the boundary.

The optimization task for analog AFCS can be solved analytically, while for digital AFCS only numerically. Natural limitation which confines the application of analog AFCS is the time delay introduced by the reverse channel. The necessary information is sent back from the receiver (BS) to the transmitter (TU) with some latency. For this reason, both the analogue and digital AFCS can be used for short-range communications like wireless or optical sensor networks, home communications, pico-cells and femto-cells, intelligent houses, RFID, electronic keys, Bluetooth, IRDA, and the like. The only exception is a distribution system (usually fiber optic) for atomic clock signals, where ultimately stable frequency and phase is distributed. The latency is not so important in these adaptive systems. The very favorable features of analog AFCS are: absence of ADC coding units (increasingly complex, expensive, and energy consuming), possibility to work with transmitters of minimal power.

Plastic Optical Fibers for FTTH and LAN: Plastic optical fibers are increasingly frequently used in FTTH systems. Plastic optical fibers for these purposes are systematically characterized by a team from IMiO (dr R. Piramidowicz). Access networks evolve from copper wires to FTTH. FTTH omits street cabinets and ADSL/VDSL/UTP lines to the end user. While FTTx has a limit around $160 \mathrm{Mb} / \mathrm{s}$, the FTTH has practically no limit, or the rate may go as high as up to $10 \mathrm{~Gb} / \mathrm{s}$. Home area network includes: home control and security, multiple 3D HDTV and VoD, security cameras, home working, internet browsing, IPTV and VoD, and video conferencing. Summing this up gives a few Gbps as a required aggregated throughput. Application of silica fibers in optical access networks has the following advantages: it is a mature technology, low attenuation, ease of integration with existing core network; and the following disadvantages: there are problems with indoor wiring due to limited bending radius, problems with network reconfiguration - specialized equipment is required, high price of active components working with SMF. The advantages of MM POF (multi mode plastic optical fibers) are: they have excellent mechanical properties, they feature user reconfigurability, low system price, low cost active components, operation in the visible; while the disadvantages are: high propagation losses, high modal dispersion. The areas of applications of POF in small specialized LANs are: home areas, data communication, automotive, object LANs, 
automation, sensing networks. There were used several fiber for tests, among them Mitsubishi Rayon Eska Premier, Mega and Super, step index, large diameter. The key parameters were measured: attenuation, dispersion and bending losses. The main loss factors are: intrinsic - absorption and Rayleigh scattering, and extrinsic - structural imperfections, pollutants, and perturbations in geometry. Dispersion has the following components: modal, chromatic, waveguide and material. The fibers were prepared for testing by: cutting with a razor blade, mounting FC connector, polishing using decreasingly rough polishing paper from $9 \mu \mathrm{m}$ to $0.3 \mu \mathrm{m}$. Attenuation was measured with cut back technique with the use of calibrated light source and OSA (optical spectrum analyzer). The fibers had transmission window (with attenuation below $0.2 \mathrm{~dB} / \mathrm{m}$ ) in the visible $400-600 \mathrm{~nm}$ and around $650 \mathrm{~nm}$. Mode scrambler was used for measurements. The fibers showed no bending losses up till $30 \mathrm{~mm}$ of bending radius. 1 and $3 \mathrm{~dB}$ of bending losses were for $20 \mathrm{~mm}$ and around $10 \mathrm{~mm}$ bending radius (one full 360 o turn) respectively. The measurements were done for the spectral region spanning 385nm-940nm. Dispersion was measured with picosecond light source and calibrated precision delay unit. The bandwidth was in the range of $100-200 \mathrm{MHz}^{*} 100 \mathrm{~m}$ for $650 \mathrm{~nm}$ without mode scrambler and 40-60 MHz*100m with scrambler. Launching conditions are critical for bandwidth measurements. The measurements can not be done without the mode scrambler. Fiber end quality strongly influences the measurement results. The POFs are good enough for FTTH systems - low cost home LANs and short distance, high speed data transmission. Further improvements of POFs is possible by micro-structuring. Microstrutural POFs are designed by the IMiO Fiber Optic team. The advantages of mPOFs are: endlessly single mode operation, extremely high modal areas, high numerical apertures, lowering of macro-bending sensitivity and propagation losses. The mPOF designed have limited number of guided modes, are bending resistant, have relatively large modal field and are optimized for propagation losses.

\section{Multimedia Technologies}

Multimedia streaming technology is researched for increasing efficiency and quality as well as reliability, decreasing costs and data rates - by using novel compression techniques. A research group of multimedia technologies under the lead of prof. W. Skarbek (IRE, PW) is working on advanced methods of image and audio processing and multimedia streams delivery. Huffman coding (code-blocks HCB) is used in the AAC. A few relevant architectures are investigated for processing efficiency. The bit rate and system requirements and then parameters were evaluated. The system was synthesized using a relevant FPGA circuit (G. Brzuchalski). Also other new algorithms are efficiently implemented in Fpga. Mpeg-TS defines container for transmission and storage of audio and video, provides basic error correction and synchronization features, and is applied in digital TV and blue-ray discs. Transport stream syntax is as follows: split to packets of 188 bytes, packet begins with a synchronization byte and header, payload is max 184 bytes, Adaptation Field may contain PCR
- program clock reference. PSI - program specific information (Program definition) includes: PAT - program association table, PMT - program map table, PID - packet ID. PES - packetized elementary stream includes: AVD - audio and video data, PTS/PDS - presentation and decoding time, CRC - checking for correctness. System architecture is composed of: $\mu \mathrm{C}$ connected via EPI (external peripheral interface) to MPEG-TS demux, (PAT, stream, PMT, PES) parsers, memory, H264/AVC decoder, AAC decoder, video/audio DAC. The input is Ethernet. The output are CVBS and TRS signals. Demux and decoders are implemented in Fpga. Transport stream and configuration data is supplied to the Fpga by a microcontroller trough EPI. The data is transmitted to the $\mu \mathrm{C}$ via Ethernet, using the UDP (user datagram protocol). $\mu \mathrm{C}$ is responsible for the translation of text commands into appropriate configuration operations. The designed, configurable, expandable system of flexible structure (A. Abramowski) is able to receive and process stream in real time. The research work also covers bit rate estimation for P-frames in the rho domain (M. Wieczorek).

A subject of research are stereo images. Super-pixel technology was applied for matching of stereo images (M. Roszkowski). The reason to choose super-pixels is as follows: a reduction of image resolution is often desirable for decrease in calculation effort and shortening time of processing; the reduction should take into account local image characteristics; the information about the objects present in the image should not be lost; image pyramid does not always meet this requirement. SLIC superpixels (simple linear iterative clustering) are relatively easy to calculate; easy to set and change the number of super-pixels in the image; superpixels centers form quite regular grid; superpixels frequently respect object boundaries. Enforcing super-pixel spatial coherence requires additional calculation step. SLIC superpixel calculation algorithms includes the steps: create new rectangular grid by evenly sampling the original image grid; distance between the grid points defines superpixel size; perform k-means algorithm locally in the image; allow the pixels to be assigned only to the superpixels, which centers are not more than superpixel size away. Local stereo algorithm is based on calculation of disparity cost volume function. Fast local image segmentation can help to decrease stereo algorithm complexity. The number of searched disparities in the local stereo algorithm is significantly reduced. The reduced disparity does not have an adverse effect on the quality of a computed disparity map.

\section{CRYPTOGRAPHY}

Distributed measurement and control networks require some form of security. Wide distribution of a network gathering sensitive data via unsecured communication channels makes it susceptible to attacks. A cryptographically secure hardware random number generator dedicated for such networks is researched by a team lead by prof. W. Winiecki at IRE PW. Justification on the safety research of distributed measurement and control systems is: they are characterized in large asymmetry of computing power, narrow bandwidth of radio communication, wide bandwidth of photonic communication, a little power supply from battery or energy harvesting, RF 
wireless or open optical communication channel susceptible to eavesdropping, intentional modification of data, interference, etc. There is a need for secure communication sub-system in these networks. The designed system is based on random number generator, as they are used in most cryptographic systems. True random number generator (TRNG) is based on the unpredictable physical phenomena. Pseudo random number generator (PRNG) is based on deterministic computational algorithms. The system was realized on chaotic generator using Atmega processor and Python software (P. Czernik, IRE PW). RDieHarder random number generator testing suite with GNU R interface was used. The realized TRNG was checked to be satisfactory solution for the measurement systems with asymmetric resource. Better randomness was achieved at a lower sampling frequency. Random bits in the 10-bit sample word are unevenly distributed. The effect of the DSP on the resulting randomness was measured.

Public key cryptography in sensor networks of unsecured communication channels, with large number of elements, should be characterized by: scalability, flexibility and ease of maintenance, low maintenance costs, less protocol overheads, larger keys than in the case of private key cryptography, simple key distribution. The following public key algorithm operations are used: one-way trapdoor functions with integer factorization problem or discrete logarithm problem; finite field arithmetic with primary fields $\mathrm{GF}(p)$ (RSA, DSA), and binary fields $\mathrm{GF}\left(2^{m}\right)$ (ECC, HECC); modular operations addition/subtraction, multiplication, and inverse computation. Possible solutions to modular multiplication $\mathrm{GF}(p)$ are: multiplication followed by reduction including multiplication, naïve reduction by division, fast Karatsuba-Offman multiplication and Barrett reduction; multiplication interleaved with reduction - Montgomery and interleaved multiplications; bit-serial versus bit-parallel architectures. The system was implemented in vhdl (J. Olszyna, IRE PW) and estimated/simulated by Gezel - power estimation; ModelSim - functional and timing simulation; PowerCompiler - power consumption estimation. The goal was to be below $100 \mu \mathrm{W}$ with power consumption to enable power harvesting units. Implemented algorithms can serve as building blocks for RSA cryptosystem. The interleaved multiplier architecture seems to be well suited for low power cryptographic applications. A possible application of the architecture is acceleration of public key primitives in constrained environments like sensor networks.

\section{Biomedical, High Performance And DNA COMPUTING}

Artificial Intelligence for Robotics: The team of Artificial Intelligence by dr S. Jankowski of ISE WUT is working on hardware-software co-development serving for advanced applications of photonic and electronic systems in robotic vehicles. AURA is a system designed for automatic obstacle avoidance in robotics using advanced neural algorithms. The system is placed on hardware based platform using multiprocessor structure. DSP functions are performed by FPGA. The system uses miniature microwave radars as sensors. The software structure is as follows. Data acquisition from peripherals is done by RDP, CRW and AHRS, which is a real time process. Topology of neighborhood reconstruction is using the perception map. Potential obstacle avoidance decision is using neural algorithm. Dynamic identification is relying on reaction to the input values via a learning procedure.

Artificial Intelligence for Unmanned Aerial Vehicles UAV: Perception and decision systems for autonomous UAV (unmanned aerial vehicles) flight are subject to intense cooperative research at WUT and at La Sapienza Uni. Roma. The research project consists of the feasibility study, design, prototyping and testing of new systems for autonomous flight of commercial UAVs for civil applications. The system includes relevant set of sensors, computer vision, data fusion as perception, and automatic decision algorithms. All of these components have to be embedded on a specific platform. The UAVs are classified as: large size - weight $>200 \mathrm{~kg}$, operating time $>24 \mathrm{~h}$, range $>1500 \mathrm{~km}$, high altitude; medium and small size - weight $5-50 \mathrm{~kg}$, operating time $5-24 \mathrm{~h}$, range $<300 \mathrm{~km}$, altitude high/medium; micro - weight $<5 \mathrm{~kg}$, operating time $<5 \mathrm{~h}$, range $<1 \mathrm{~km}$, low altitude. Automatic navigation means that the UAV has an electronic Autopilot System (AP) with a preloaded Flight Plan (FP). It permits: auto take-off, cruise following waypoint, auto-landing. Autonomous flight means that the UAV has an electronic/photonic system that permits the "Sense and Avoid" operation which includes: detection of obstacles (fixed or moving, in air or in the ground, cooperative or not), computation of Time to Contact, processing and elaboration of an escape strategy. UAV with large payload (over 100kg) have large power source onboard, high processing capabilities of electronic system and large space in the avionic bay. They permit an efficient use of data fusion from multispectral cameras and extended, heavy radar systems. UAV with medium and small payload (less than 20kg) have poor power source available onboard, quite low processing capabilities and little space in the avionic bay. The most frequently used technology in such a case relies on the Optical Flow (OF) solution for motion detection computed from the real time vide streaming and acquired from cameras installed onboard. The system is based on bio-inspired algorithms, like the vision system of a fly. Data sources are GPS, IMU, Laser Radar (Lidar), bio-inspired machine vision, data processing algorithms. All the data are subject to data fusion with the output enabling autonomous flight. The Optical Flow technology is defined as a pattern of apparent motion of objects, surface and edges in a visual scene caused by the relative motion between an observer and the scene. The OF scene analysis algorithm relies mainly on the brightness changes of each pixel in the frame sequence. The OF divergence activates the saccade. The insect flies in the direction of the greater OF. The computation cost for OF has to be decreased by dividing the frame to subframes and optimizing the calculations.

DNA Computing: The work on DNA computing is done in WUT and WULS (Warsaw University of Life Sciences) cooperation. One of the intermediate aims is to master the genome browser algorithm. The Genome Sequencing Project is the world's largest action of producing the full dataset to create maps of genomes. The recognized number of genomics sequences ATCG is continuously increasing. The aim of the GBA system is to display genomic features fast and in an 
easy way. The research procedures go from acquisition of the genomic data to the genome draft, which is partial assembled genome, and include the following steps: short reads - sequencer output; contigs (physical map of the genome that is used to guide sequencing and assembly) - sequencer output assembly; BES - back end sequences; scaffolds - contigs and BES assembly; markers map - known sequences and position on chromosomes; chromosomes - with assigned all elements; genome draft. Genome annotation, which is a process of attaching the information to the structures, concerns two processes - structural and functional. Structural annotation consists of the identification of genomic elements: gene-like structures, coding regions, location of regulatory motifs. Functional annotation consists of attaching biological information to genomic elements: biochemical function, biological function, involved regulation and expression of interactions. The genomic data are stored in a standardized very large tabular format, imposed by classical biological tradition, and very difficult for further digital processing by ICT systems. New approach is required for "DNA computing" which employs high throughput assays, automatic operator-less processing, advanced statistics and smart bioinformatics. All these features have to be combined in a single smart display of the genome overview - a genome user-friendly browser. The information display goes from a single nucleotide to a chromosome with annotated data, from multiple and diverse sources, including gene prediction and structure, proteins, expressions, regulation, variations, etc. The example is the MSU Rice Genome Annotation Project (GBrowse). The advantages of GBrowse are: simple and clear view of the genome structure and detailed information of genes, easy accessible data, improvement of the usage of knowledge stored in genome. The disadvantages of GBrowse are: installing, configuring and updating is difficult, input of new genome data takes very long time, there are used scripting languages. The GenWeb - a new genome browser. Which is a joint effort of WUT and WULS introduces new facilities in algorithms, data structure and in software architecture. The Intervals contain: reads, contigs, scaffolds, structural and functional annotations data. The Intervals tree node contains a point in sequences (index) and intervals with common index. Software bases on client-server solution. The client uses web browser. There are used the following programming languages and libraries: $\mathrm{C}++$ (calculation library), Python, Adobe Flex, DJango, BioPython (access to external databases), Boost and others. The system has access to NCBI and EBI databases. The value of genome sequences lies in their assembly and annotation, but its practical usefulness depends on the way of management and presentation.

There are researched different methods of decoding gene sequences. The aim is to lower the costs, shorten the sequencing time, increase the accuracy and generally make the method accessible for wide medical procedures. Proteins are linear sequences of amino acids. There are at least 20 amino acids including Alanine, Leucine, Serine (A,L.S,...). DNA is a double helix of nucleotides - nucleic acids (C T A G) plus $\mathrm{U}$, instead of T mainly in RNA. Single strand RNA contains DNA information out of the nucleus. Peptide biosynthesis goes through DNA - mRNA, Rybosome plus tRNA plus rRNA, which results in polypeptide. Codon is a triplet of DNA, eg. GCA, ACT, CGT,... There are 64 codons, and each peptide is coded by at least one codon in 1 to $\mathrm{N}$ relation. Codons have specific frequencies in different proteins. The decoding algorithms base on codon frequencies. The approach is: choose a codon randomly, select the most frequent codon in a given organism, randomize codon with respect to its frequency in the organism, optimize locally in sliding window. The properties of such methods are: require the codon frequency table, low expression for the resulting synthetic gene, tertiary structure (folding), biosynthesis is stopped with too low level of corresponding tRNA, do not reflect any grammar. Hidden Markov Model (HMM) is used successfully for decoding of gene sequence. Markov model is a stochastic model that assumes Markov property of the researched system defined as: Q finite set of states A, C, T, G; probability S of start states equal to 1; transition matrix A of unitary properties. At these assumptions the probability of getting sequence $s$ is described analytically as a product of elementary conditional probabilities. Hidden Markov Model is somewhat more complicated, because the entire state is not visible, or there is an additional hidden level. The HMM has in this case the following arguments: V observations (20 amino acids); $\mathrm{Q}$ - hidden states (64 codons); A - transition probability matrix, E - emission probability matrix; $S$ - start probability vector. Probability of getting an assumed sequence is also a product individual probabilities from emission and transition matrices. HMM tends to reflect frequency table and can model grammar. The task is, thus, to get hidden sequence knowing the output sequence. Viterbi learning/training algorithm is applied for back reflection. Out of the sequence $\mathrm{Q}, \mathrm{V}, \mathrm{E}, \mathrm{A}, \mathrm{S}$, there are two parameters one can change - start probability vector $S$ and transition probability matrix A. Assuming that in the current algorithm $\mathrm{S}$ is constant and equal to $\mathrm{S}\left(q_{i}\right)=1 / 64$, and the probability matrix is constant. The key factors in the algorithm success are: fast Viterbi implementation, efficient optimization algorithm, more complicated mode - higher level HMM with additional loops in Viterbi, adding folding and other effects, application of neural networks.

Classification of DNA strings for DNA recognition and computing: One of the research directions on DNA is creation of a simple and effective classifier for strings (dr S. Jankowski ISE WUT). Feature selection was applied with Contrast Pattern Kernel (CPK) for Phosphorylation prediction. In the real life problems, many data is represented as strings. For example, in bioinformatics, strings are used to represent DNA or amino acid sequences. Some of the most effective classifiers like kernel methods, particularly SVM, require the input data to bee represented as real number vectors. How, in such a case, strings can be represented? There are introduced, as a solution, the string kernels, which are special functions designed for maintaining string data. A kernel function is often interpreted as a measure of similarity in a sense that the kernel has a large value when the arguments of the function are similar. For example, two biological sequences will be considered similar if they have a good fit. Any sequence consists of smaller sub-sequences (single symbols, pairs of symbols, etc). We can represent a sequence by substrings 
appearing in it. Two sequences will be considered similar if they share many common substrings. Spectrum kernel is one of the most popular string kernels. This kernel describes the sequence $\mathrm{x}$ by the frequency of occurrence of all possible substrings a of constant length 1 , created over alphabet S. The kernel is defined as a dot product or RBF function between the two sequences represented in the described way. The task to be solved is: substrings of what length should be used? If we have so many features, substrings for the spectrum kernel, it is essential to select the most relevant substrings. Thus, the basic idea is instead of creating a vector consisting of all possible substrings, one can use contrast patterns. A contrast pattern is a substring that is common for one class and rare for the another one. More, one should not consider a complete sequence but select the most relevant position in the sequence. The most important attributes in a sequence with respect to each class can be selected by using the classification tree. The tests were carried out for the input data set containing 17-symbol amino acids sequences grouped with respect to their reactions with several selected enzymes (kinases). They catalyze the reaction of phosphorylation for selected sequences. Phosphorylation activates or deactivates many protein enzymes, causing or preventing the mechanisms of diseases such as cancer and diabetes. The least-square support vector classifiers have been trained and tested. Thanks to the feature selection one can obtain more effective and simpler classifiers.

Haplotype frequency estimation: Different types of heuristics is researched for haplotype frequency estimation with large number of analyzed loci. The research is carried in a group lead by prof. L. Mulawka at ISE PW. Haplotype is a combination of alleles at adjacent loci on the chromosome that are transmitted together. Genotype is a combination of alleles the individual carries. Polymorphism is an occurrence of two or more alleles at the same single locus. Determining haplotypes with laboratory methods is expensive and time consuming. In contrast, there are many cost-effective techniques for determining genotypes. In general, it could be impossible to infer haplotypes from genotype data. There are different strategies for inferring haplotypes. Phasing problem is solved by: pure parsimony, hidden Markov model and other Bayesian approaches, and maximum likelihood estimates. The problem is complex since the number of haplotype resolutions grows exponentially with number of observed loci. Every algorithm employing full space search would operate with very high complexity. This is why it cannot be directly applied to phasing long genotypes. Genotypes can be divided into shorter pieces that overlap. Piece length is fixed, so is computation time. Phasing fixed number of pieces has lower complexity. Multiple pieces can be phased in parallel. If phasing algorithm is convergent, the total error should not be large. The error and execution time are function of width and overlap parameters. Hardy-Weinberg Equilibrium states that allele and genotype frequencies in a population remain constant.

Classification of RNA secondary structures: Prediction and classification of RNA secondary structure is done using such algorithms as: Naïve Bayesian Classifier (NBC), Decision Tree Classifier (DTC), and k-nearest Neighbor Classifier (kNNA).
The aim is to optimize the algorithms against simplicity, calculation time and cost (R. Nowak, ISE WUT). The secondary structures are: hairpin loop, internal loop, bulge and junction. RNA secondary structures can be generated by RNAstructure application. MBC is based on Bayes theorem which concerns counting the value of the cause probability when the result is known. DTC shows considerable complexity for large data sets. kNNA relies on selection of the dominant categories in a subset of the set's training with the smallest value of distance. kNNA has known problems with noisy training data. RNA STRAND is the RNA secondary structure and analysis database which has over 3500 trusted secondary structures of 36 different types. The categories with the highest number of added secondary structures are: Transfer RNA, Transfer Messenger RNA and Ribonuclease RNA. These ones represent nearly $55 \%$ of all examples. The prospects of development of the algorithm are as follows: connect to large secondary structures database, adding secondary structure predicting module implemented using different algorithm, allow to create user accounts, and storing the results of classification in a database.

Stem cell therapy: Design guidelines of quality assessment model for stem cell (SC) therapy is researched by a collaboration of WUT, WF-UW, ICH Warsaw. There are three basic types of stem cells, relevant to their potency: totipotent (differentiate into anything), pluripotent (all except placenta), multipotent (replenish skin and blood cells). SC are from umbilical cord blood - USC, bone marrow - BMSC and embrional - ESC. USC are beyond dispute concerning the ethicity, as opposed to ESC. USC have greater potency than BMSC due their younger age. USC are classified as hematopoetic HCS, endothelial progenitor EPC, mesenchymal MSC, unrestricted somatic USSC and very small embryonic like VSEL. The quality model bases on linear relation between endogenic variable (therapy quality) and the exogenic variables (effectiveness, efficiency, cost, time, etc).The work on the model goes along the following path: methodology evaluation, data source choice, data cleaning, parameter selection, model verification/validation. Conceptual and graphical resources are taken from MEDAFAR - classification of pharmaco therapeutic referrals.

Analysis of human gait cycle for robotics and medicine: The research on human gait cycle is done using photonic and video sequence analyses (dr J. Dusza of WUT and Valencia University). A simplified model of mean double step (MDS) for gait cycle in human body movement was introduced. Human gait is defined as bipedal, biphasic forward propulsion of centre of gravity of human body, in which there is alternate sinuous movements of different segments of the body with least expenditure of energy. Gait cycle can be precisely recorded by multi-video system with multiple markers. The gait cycle contains: right initial contact, left preswing, eft initial contact, right pre-swing. The support phases are: double support, right single support, double support, left single support. Right stance phase is simultaneous partly with left swing phase and vice versa. The following parameters are measured using kinematic methods (by at least 4 cameras, with reflective markers glued to the body in standardized positions): coordinates of selected points (markers) vs. time, angles vs. 
time (Left and Right - L and R, hip, knee, ankle, pelvic), trajectory of selected points, cyclograms, bilateral cyclograms, velocity, acceleration, joint rotation. At least six degrees-offreedom marker set for gait analysis is established including: L and R, Sagittal, Coronal and Transverse. The global measured parameters are gait length and duration of gait. These two parameters create a circular area for many measurements, in the global parameters plane. The used hardware is fast multichannel frame-grabber. Reconstruction of $3 \mathrm{D}$ is done with epipolar techniques. The current research problems are: can be the movement described by simple dependencies; parametric model of gait; what kind of process is stable gait; understanding the asymmetry in gait; and gait model simplifications. Analysis of the functions show that the gait is not a periodic process, neither it is an almost periodic process. Walking is a cyclical but not periodic process. And there is no simple mathematical analytical definition of a cyclic signal. The closest mathematical approach is to add periodic model, random component and chaotic component. The periodic model bases on MDS - Mean Double Step (J. Dusza). The analysis includes averaging an arbitrary number of recorded gait cycles in the spectral domain. This approach leads to very high data compression ration and enables efficient gait analysis, tool standardization and further research. Photonics solutions are essential in this research.

Population study of health: Data management and quality assurance in very large data sets and bases, especially medical, is a very difficult and effort consuming task. It requires rigorous standardization to be useful for further research. The data may originate from such different and nearly incomparable sources as measurements in different conditions, including image data, or from an interview. Image data may come from different sources like: X-rays, tomographs, ultrasonographs, OCT, PET, optical, IR, multispectral, etc. Different parameters from these data contribute in a different way to the final system model. A consortium of COI, IMW, NTNU - Trondheim, UW, PIS, FPZ,WUT, CC-IoO inside a Polish Norwegian PONS project (dr Z. Wawrzyniak and dr D. Paczesny, ISE PW). The reasons of PONS study are: there is a wide gap in health and disease in Poland compared to western European countries, an urgent need to understand the underlying causes of these differences, to study population with respect to important factors related to health and wellbeing, understanding of important causes of morbidity and mortality in Poland, to establish a solid knowledge base for the prevention of these major causes of premature morbidity and mortality. Data management in the system includes: abstraction, collection, transmission and storage, coding, processing, security and quality assurance. Data abstraction includes: HSQ - health status questionnaire, medical measurements, BioBank, e-collection and checklist for data consistency. Data processing is done using data mining methods. The data includes altogether around 1000 variables. Data is good when they can be used for healthcare decision support and provide accuracy and integrity. Data quality assurance is a dynamic and systematic process of profiling the data to discover inconsistencies, and other anomalies in the data and performing data cleaning activities (removing outliers, missing data interpretation) to improve the data quality. The QA consists of the following steps: determine the data items causing problems, profile the offending data, determine what the data should be, determine how to finally clean the data. QA process consists of three phases: predata collection, data collection and management, and post-data collection. The use of modern IT, communication infrastructure, advanced database technologies and remote software and hardware structures efficiently support the research of massive epidemiological surveys.

Pneumography: Automatic breathing measurement systems may base on spirometry, optical methods, electrical methods, or combined ones, etc. Impedance pneumography is based on relevant measurements performed on a patient's body. Ambulatory impedance pneumography is researched in Faculty of Mechatronics at WUT (M. Mlynczak, G. Cybulski). In clinical stationary conditions several methods to measure respirations may be used, which are completely unsuitable for ambulatory conditions. On the other hand there are many clinical and physiological reasons for requiring a reliable, long-lasting quantitative measure of respiration parameters. Impedance pneumography (IPG) seems to be a suitable technique satisfying the mentioned requirements. Long term respiratory monitoring is possible, at low cost and relatively easy to use without active cooperation of the patient. IPG measures changes in trans-thoracic electrical impedance with electrodes fastened to patients skin. There are two adding effects to the increase of impedance during inspiration: gas/fluid volume rate and conductance paths are increasing. The change is in the range $0.02--0.2 \%$, but the measurement conditions are very noisy. The measurement system requires not only repeated calibration but also complex noise removal. There are many sources of noises: physiological processes, heart beating, patient movements, etc. One of the measurement methods may base on injecting RF signal into patients body and measure modulation of this signal by physiological processes, including breathing, and then filter out other side modulations and noises. Also a multisensory method may be applied, with sensor signal fusion. The system is predicted for regular ambulatory practice but also for sport exercising and for augmenting the treatment of asthma and functional respiratory disorders.

\section{BRAIN - COMPUTER INTERFACE}

Brain computer interface BCI is an innovative biomedical technology that use brain activity to control computer environment. The computer, in turn, may be used to control other systems and/or devices. The neuro-feedback systems available are: BrainGate - invasive neural interfacing to help disabled people; g.Tec Guger Tech. - noninvasive biopotential measurement systems; Emotiv Epoc - BCI device with multiple electrodes for home use; NeuroSky MindWave simple neuroffedback; OpenEEG - the most popular neurofeedback open source platform, and other with practically no support like BrainBay, NeuralServer, EegMir, etc. From engineering point of view the BCI is a versatile platform for biological signal acquisition, processing and presentation, 
targeted at electrical brain activity. The basic features of the $\mathrm{BCI}$ as researched today are: can use invasive or noninvasive methods to acquire brain and/or other physiological signals, including photonic, RF, mechanical, magnetic and other; can use different methods of brain activity measurement like EEG, fMRI; no signal input into brain except safe audio-visual stimuli, complex analog measurements system working with ultra-low-voltage biological signals; advanced digital structure for signal acquisition, processing and decision making; use neuro-feedback mechanisms for closed-loop control. A research work on BCI is carried out in the Biomedical Engineering Research Group (ISE WUT) lead by prof. A. Grzanka (T. Cedro, K. Chojnowski, J. Frączek, L. Czupryniak). The assumptions for the system design are: low-cost fully featured BCI system; modular construction for easier experimentation and prototyping; is based on open source and free software as development platform and user environment; overcome existing low-cost system limitations and bring new features; aim at a new standard for academic and home use of the BCI. Measurement front end of the system acquires the EEG signal, of the amplitude $5-50 \mu \mathrm{V}$ and frequency range $0-100 \mathrm{~Hz}-$ with delta, theta, alpha, beta and gamma waves - of the following frequencies respectively: $1-4 \mathrm{~Hz}, 4-8 \mathrm{~Hz}, 8-12 \mathrm{~Hz}, 12-40 \mathrm{~Hz}$ and over $40 \mathrm{~Hz}$. The measurement issues are: low signal amplitude, high noise level and high skin impedance. Thus, the hardware has to be quite refined. The EEG was analyzed in real time to extract the signals responsible for simple functions like eye close and open, hand and leg movements etc. The BCI hardware uses new fully featured BCIOP protocol (BCI open protocol) basing on TLV mechanism (tag length value). BCIOP features are: exchange data between target equipment and host PC; allow real-time applications with low latency and high throughput; control functions and parameters of target device; storage measurement results and off-line analysis; implementation of user interaction and realization of nonstandard research procedures; have library form to be linked into small footprint firmware and full featured host software; easily adapt to hardware specific needs such as data format, sampling speed or time intervals, and others.

\section{Ontological Databases, Languages, Logics, MACHINE LEARNING AND ARTIFICIAL INTELLIGENCE}

Ontological approach to the development of some ICT technologies leads to deeper understanding of large and complex systems, which are generally very difficult to be described in a formal algorithmic way. These systems include, worlds, countries, societies, large corporations and institutions, etc, but also very large technical and civilization infrastructures, like research, medicine, communication, transportation, environment, global and local safety, large events, etc. The components of the more formal description trials are: different kinds of logics and logical systems, descriptive languages, knowledge analysis, notions definitions, automated methods of building models and concepts in logical systems, checking the systems for inconsistency, building inherence paths, reasoning methods, evaluating sets of theses, checking the assumptions, presenting use cases, and many others.
Epistemic logic and its relations to the modern ICT was a subject of Wilga topical session prepared in cooperation by prof. J. Mulawka (WUT) with the research team of prof. E. Nieznański (UKSW). Ontological and epistemic approach to future photonic and electronic systems, in hardware and in software spaces, turns out now to be an essential development basis of the intelligent virtual worlds. Hardware and software/firmware is a sort of a basic foundation of such systems. Future development of photonic/electronic systems building fully intelligent virtual worlds is expected to base on more sophisticated ontological base than it is today. The basic features of such worlds in the future will be very probably full conscience of subjects and objects, and full submergence. Today, strictly speaking, the used ontology is simplified, if not primitive. The search for new extended bases for artificial intelligence systems go into various directions. Some of them are: new logic systems, epistemic logic, research in knowledge representation, formal description of ontological systems, and others. A logic is a formal language which supports reasoning - natural and artificial. The logic has precisely defined syntax and semantics. The logic is fully independent of the domain of application. As a consequence, representation of some things in logic may not be very natural. Epistemic logic is a subfield of modal logic concerned with reasoning about the knowledge. It was rediscovered in modern form by Kripke in 1963. There are four basic modal operators in epistemic logic, which may be digitized and applied in a digital world: $\mathrm{W}$ - one knows that; $\mathrm{S}$ - one is certain that, $\mathrm{P}$ - one supposes that, $\mathrm{D}$ - one admits that. The logic defines/induces relations between the operators, and bases on coherent axioms and rules, for example analogous to CPL - classical propositional logic: A1. $\mathrm{S}(p \rightarrow q) \rightarrow(\mathrm{S} p \rightarrow \mathrm{S} q)$; A2. $\mathrm{S} p \rightarrow \mathrm{SS} p$; Df.W:W $p \leftrightarrow \mathrm{S} p \wedge p$; A3. W $p \rightarrow \mathrm{SW} p$; A4. $\mathrm{S} p \rightarrow \mathrm{P} p$; Df.D: $p \leftrightarrow \sim \mathrm{S} \sim p$; A5. $\mathrm{P} p \rightarrow \mathrm{D} p$. Using such operators and relations between them it is possible to prove theses. The used axiomatic method involves replacing a coherent body of propositions (like a mathematical theory) by a simpler collection of propositions (like axioms). The axioms are designed in such a way that the original theory can be deduced from the axioms, using among others modus ponens rule, semantic tables of Beth, literals, clauses, clause sets, resolution rule. The general goal is to adapt and test the above methods into epistemic logic, which may require research on new theorems, and to incorporate epistemic logic into the ITC engineering knowledge.

Virtual intelligent worlds are dynamic entities. Dynamic epistemic logic embraces such fields as: epistemic logic, public announcement logic, action models, expressivity and probability. Dynamic epistemic logic is the logic of knowledge change. DEL embraces by no means a single logical system. It is an overhead for the whole family of logical systems. DEL is expected to allow the artificial intelligence to specify static and dynamic aspects of multi-agent systems. The language of DEL bases on dynamic semantics, which is a subarea of semantics. It distinguishes between de dicto and de re understanding of sentences. It is concerned with the change of information and the change in general. The main idea of dynamic semantics, in information theory and artificial intelligence sense, is that the 
meaning of a syntactic unit, irrespectively if it is a sentence of a natural language or preferably a computer program, is best described as the change it brings about in the state of a human being or a computer. The meaningful information in such an ontology is regarded as an entity (existence) that is in functional relation to a particular subject. The subject has a certain perspective in the real or/and virtual world. Such a subject is defined as an agent. Epistemic logic trying to describe the general relations of an agent may be written formally: I know that $p\left[\mathrm{~K}_{a} p\right]$. He does not know that $p\left[\neg \mathrm{K}_{b} p\right]$. He knows whether $p\left[\mathrm{~K}_{b} p \mathrm{~K}_{b} \neg p\right]$. He knows that I know that she does not know that $p\left[\mathrm{~K}_{b} \mathrm{~K}_{a} \neg \mathrm{K}_{c} p\right]$. Epistemic models use A. Kripke structures $\mathrm{M}=(\mathrm{S} ; \mathrm{R} ; \mathrm{V})$, where $\mathrm{S}$ is a nonempty set of states, $\mathrm{R}$ gives an accessibility relation $\mathrm{R}_{a} \leq \mathrm{SxS}$ for every a belonging to $\mathrm{A} ; \mathrm{V}: \mathrm{P} \rightarrow \rho(\mathrm{S})$. If all relations $\mathrm{R}_{a}$ in $\mathrm{M}$ are equivalence relation, we call $\mathrm{M}$ an epistemic model. Public announcement logic, important in virtual reality, bases on the following statements. After announced that $p$, everyone knows that $p[(p) \mathrm{E} p]$. After it I announced that $p$, it is common knowledge that $p[(p) \mathrm{C} p]$. Example: After it is announced that none of the programmers know the newest programming language, all the programmers who do not know this language know that they do not know this language $\left[\left(\Lambda_{a \in A} \neg \mathrm{K}_{a} m_{a}\right) \Lambda_{a \in b} \quad \mathrm{~K}_{a} m_{a}\right]$. The epistemic modeling of a real and/or virtual situation contains the following components. Given is a description of a situation (for example in the Internet based virtual reality). The designer/modeler tries to determine: a set of relevant prepositions, a set of relevant agents, a set of states, a nondistinguishability relation over these worlds for each agent action models. Further step in creation of an intelligent virtual world is a dynamic modeling. There is given a description of a situation and an event that takes place in this situation. The designer/modeler first models the epistemic situation, and then tries to determine: a set of possible events, preconditions for these events (for example space and time coordinates, but not only, also more complicated ones), a nondistinguishability relation over these events for each agent. An action model M, in such intelligent virtual reality, is a structure over a finite domain of action points or events $S$, maintaining an equivalence relation on $S$, denoted by operator $\sim_{a}$, and fulfilling precondition function pre: $\mathrm{S} \rightarrow \mathrm{L}$ that assigns a precondition to each $s \in \mathrm{S}$.

Building of practical intelligent virtual environments (very complex intelligent software systems basing on rich ontology, we say ontology driven) requires the ability to represent the knowledge. Knowledge is defined here simply as all what I know, and is a symbolic description of the real world. It is a set of messages from a specific area. The task of knowledge representation is split to categorial objects representation. The applications of categorial object representation are: categorial LISP, PROLOG, semantic networks, implementation of machine engines in extended logics, and logics of categorial objects, concepts and systems. The most objective (and popular in relevant research) Knowledge Representation Methods (KRM) are independent of implementation. They base on procedural representation (called production rules) and may contain simple unification processes and could be used as tensors of knowledge. KRM is a root of Semantic Networks (now the Semantic Web) in the original linguistic M. Ross Quillian and Allan M. Collins sense (semantic knowledge representation). Semantic network connects all possible notions combined with an object or subject of the knowledge. This may include: description, varieties, features, actions, relations, applications, linkages, answers to such questions like: it has, it is, made of, used for, where, why, what, etc. Modified Quillian approach to KRM includes: simple and perceptive ways to represent information, object oriented approach to knowledge representation, natural representation, etc. However, yet the meaning of semantic networks is not always well defined. Categorial Semantic Networks include the following categories: $\mathrm{T}$ - things, $\mathrm{F}$ - features, $\mathrm{S}$ - situations, and $\mathrm{C}-$ classes. The categories are related by the following operators: Relatives $\mathrm{Y} / \mathrm{X}=\mathrm{Y}$ of $\mathrm{X}$, relation $\mathrm{T} / \mathrm{T}$; Concatenations $\mathrm{X}^{\wedge} \mathrm{Y}$ $=\mathrm{XY}$, relation $\mathrm{F}^{\wedge} \mathrm{T}$; Categories $\mathrm{X}, \mathrm{X} 1, \ldots, \mathrm{Y}, \mathrm{Y} 1, \ldots$ categories $\mathrm{T}, \mathrm{F}, \mathrm{S}, \mathrm{C}$; Hypostasis $\mathrm{Y} / / \mathrm{X}=\mathrm{Y}$ arising from $\mathrm{X}, \mathrm{F} / \mathrm{S}$ being $\mathrm{X}, \mathrm{T} / \mathrm{C}$ aggregate unit from the class; Operators of relation and quantity: of, from, $\wedge, \sim,=$, a, the, very, some, all. Using these formal tools any expression and then clause in Categorial Networks is analyzed as a succession of operators QTFSCA. Formal tools include categorial Lisp and Prolog. Lisp consists of objects - entities. An object is composed on n cells. Numerable cells represent categories. Classical Prolog variables represent everything. During unification category everything is substituted by formal variables. To infer a method of resolution is used, which creates a very big solution space. In extended Prolog, oriented for semantic networks, objects are represented by cells with categories. During the unification only the adequate categories are matched, and again a method of resolution is used to infer. As a result a huge space of possible solutions is significantly reduced. To find a solution, much smaller space is searched, what is much faster.

Relatively simple (yet more and more complicated) examples of ontology driven virtual worlds are existing in some advanced PC games. Ontological drivers may concern separately particular layers of the virtual reality like communication, bots behavior, avatar's personality, properties of the space/nature and/or all the layers together. Ontological background concerns also the relations between the mentioned layers of the virtual reality. A lot of different techniques is applied for practical solution. One is interfacing Clojure with Pogamut platform, an AI software exercise tried at Artificial Intelligence Lab. of ISE PW. Clojure is a contemporary dialect of LISP language. It supports functional and multithreaded programming and runs in JVM and CLR. Clojure: is succinct and factorial functional language, integrates with and is embedded in Java, can be integrated with pogamut, uses Java libraries, runs as Java threads, allows for inline Java code, Clojure functions are called by Java code, and is available as a clojure.jar Java library. Pogamut, under constant upgrade, simplifies creation of bots/agents, controls squads of bots and agents in virtual environments, enables flexible bot navigation, integrates several Virtual Environments (like UT, UDK-UE, Defcon), enables simple reinforcement learning and has ontology driven communication. Squad control includes: coordination, communication, organizational structuring, maneuvers, formations, planned and emergent behavior by influence vectors and team 
controller. Learning enables gathering of experience. Ontology driven communication bases on FIPA-ACL agent communication language. Integration of Clojure-Pogamut bases on modular horizontal layered architecture (MHL) which consists of layers and mediators. The mediators can execute multiple layers. Each layer represents a simple or complex behavior and has access to agent components like memory, communication, body, behavior. Abstraction allows for simple Mediator change according to strategy design pattern. MHL architecture enables programming of each layer in a different language - Clojure, Scala, Groovy and others. Clojure code can be called from Java in three ways: a compiled jar file with class generated by gen-class; a clj script file clojure.lang.RT or inline by string object.

Modal Logics. Modal logics have been widely studied for reasoning about knowledge and belief, usually based on the monomodel logics S5 and KD45 for reasoning about knowledge and belief respectively. Both logics have standard axioms, which means that knowledge and belief both satisfy positive and negative introspection. The logic S5 also has axiom $\mathrm{T}$, which means that knowledge is veridical, while KD45 has also axiom D which mean that belief is consistent. Kripke model was applied to build an algorithm of multimodal logics of belief. Kripke model can be presented as a graph, consisting of vertices - possible worlds, edges - transitions, relations between possible worlds. The graph is complete and directed with bidirectional edges. Each edge has a transition factor which changes with time. If the transition factor is above a defined threshold, the world pointed by this edge is accessible, otherwise is not. After every event in the world, the beliefs of an agent are updated in the Kripke model by changing the transition factor. Each action taken by other agents can be seen as positive or negative. Positive actions increase transition factor, while negative actions decrease the transition factor. Transition with factors below the threshold are not updated and are considered inexistent - because the worlds pointed by them are inaccessible. The model can be efficiently implemented in a PC in server - client architecture. The presented approach may facilitate or augment building models of large and complex systems like global communications or next generation of the future Internet. Expanding Formal Languages. The research on expanding formal languages is carried on in UKSW (CSWU) University, in a group of prof. K. Świętorzecka. The language is treated as a family of n-languages which get some epistemic interpretation. The changeability is described consisting in adding new expressions to the language to which they did not belong just because an agent did not know them before so he could not consider them. A formal basis of the proposed description is the calculus LC. LC is extended by definition of two operators $\mathrm{G}^{+}$and $\mathrm{G}^{-}$. In the result the system LCG is obtained, in which one can compare two types of changes described by operators $\mathrm{C}$ and $\mathrm{G}$. $\mathrm{C}$ is an operator of change and it reads: as it changes, that. LCG calculus and LC logic has all tautologies of classical sentential logic. The introduced $\mathrm{G}$ operators may also be combined with some temporal notions. LC may be extended by an operator $\mathrm{N}$, to be read - next it is the case that... LC extended by $\mathrm{N}$ is equivalent to temporal calculus
F of Prior which has an interpretation in discrete and linear structure of time. The system is expected to be formally a basis for algorithms researching the changeability of languages.

Inconsistency in logical systems: Inconsistency is a term often associated with paradoxes or antinomies. It is a relationship between two sentences where one sentence is a negation of the other. Internally inconsistent sentence is a conjunction of two contradicted sentences or any other sentence which could be transformed into such a conjunction. The subject of research is automated proving of inconsistency by application of IT. IT can be used for proving and checking correctness of logical proofs. The implementations employ an indirect proof technique - reduction ad absurdum. An example of automated theorem proving is Prover9 software environment. The consistency cannot be proved automatically, in contrast to inconsistency which can be proved automatically. The proving bases on the assumption that any syntactically correct formula is derivable, as well as contradiction is derivable. A solution is to determine the minimal set of axioms which, when accepted all at once, bring inconsistency to the theory. Such algorithmic methods are available and are used for inconsistency checking and for redundancy checking of set of axioms (P. Orzeszek).

Automated formal concept analysis: Formal concepts analysis (FCA) is subject to normalized approach and research starting with philosophical background and ending in preparing software environments. This concerns wide range of processes spanning from social phenomena to extended and ultimately complicated communication systems. A research on these subjects are carried out at CSWU (M. Porwolik). Constituents of thought are analogous to words, which are constituents of a sentence. They are believed to be combinable. Categorization is understood as primary tools by which one determines that something falls into a category. Inference is understood as follows. When someone applies the concept to some thing (and makes categorization), someone else can use the concept to infer facts about that object. Linguistic meaning is understood as follows. It is the meanings of the words or components of the meanings. Concepts are expressed when one uses words. Concepts are not meanings but play a central role in the epistemology of language. Representations refer to things and are intermediaries between mind and world. Concepts would express: its extensions, intentions as functions and properties, and present referential points of view. An extension is a set of actual objects that satisfy the concept. An intention is a function that maps a possible world to the extension of the concept in that world. Properties are the properties these object share. FCA is data analysis based on the notion of a concept, with its components like extension and intention. Data is represented by a very basic data type and in a formal context. This trem was introduced by R. Wille in 1984. FCA applies lattice theory and order theory. The application is in data and text mining, machine learning, knowledge management, semantic web, etc. FCA is for analyzing of constituents of thought, categorization, inference, finding linguistic meaning and representation. Concepts in FCA are specified by extension and intention and can be concepts of individuals.

Inherence in logical systems: PowerLoom environment is used for implementation of inherence calculus (M. Wachulski, 
ISE WUT). Inherence calculus s the calculus built on the top of subsumption relation and inherence relation (relationship) using formal language. It operates on abstracts. Abstracts are all beings (concepts and individuals). Concrete abstracts concern only individuals. PowerLoom technology is a successor of Loom System. It has more expressive representation language, less arcane syntax, better scalability and portability and extensibility, it is based on first-order predicate logic (not description logic), and has pragmatic approach. There, the usability is more important than theoretical formalism and expressivity is more important than inferential completeness. The query engine is used to retrieve asserted and inferable statements. Prolog style backward inference is enhanced by recursive subgoal detection, proper handling of negation, hypothetical reasoning, resource bounded depth-first or iterative deepening search, proof tree (justification) recording. It features forward inference and simple constraint propagation, equity and inequity reasoning, subsumption reasoning, relation and instance classification, partial match for reasoning with incomplete information, WhyNot abductive inference for query diagnostics, and extensible reasoning specialists architecture. General idea is to put entities and relations in PowerLoom module and supply the test module with the PowerLoom results along with test data generator and queries. Power Loom was shown during tests to be a feasible environment to build rich information systems that exploit formal ontology to its maximum.

\section{Conclusions, Acknowledgments, Future}

WILGA Symposium from its beginning in the mid nineties has produced more than 2500 articles, out of which over 1000 were published in Proc.SPIE. Several hundred of them are associated with the research activities of the PERG/ELHEP Research Group at ISE WUT. The Group is an initiator and major organizer of the WILGA Symposia.

The WILGA 2012 meeting was a fruitful event gathering young researchers from the fields of photonics and electronics systems. Photonics and Web Engineering 2012, in its $30^{\text {th }}$ edition keeps the style of being a wide and friendly meeting for young researchers, mainly Ph.D. students. The Wilga Symposium proceedings are good memoirs of the development of photonics, optoelectronics, metrology and some telecom technologies in the academic laboratories in this country.

The author would like to acknowledge all the persons involved in the organization of the conference on 'Photonics and Web Engineering', Wilga 2012, in particular the Program and Organization Committees. The author also would like to thank all participants of Wilga Symposium for making the event again and again a success. This paper was prepared using the invited and contributed presentations debated during Wilga 2012. Some fragments of the text were quoted from these presentations and from session discussions

The 2013 Symposia on Photonics and Internet Engineering will be held on 26-29 January at WEiTI PW building in Warsaw and on 28.05-03.06 in Wilga Resort by PW. The organizers warmly invite young researchers to present their work. The WILGA Symposium web page is under the address: http://wilga.ise.pw.edu.pl.

\section{REFERENCES}

[1] R. S. Romaniuk and K. T. Poźniak, Eds., Wilga 2002. SPIE 5125; Photonics Applications in Astronomy, Communications, Industry, and High-Energy Physics Experiments 2002, 2003, 472 pages, 55 papers.

[2] R. S. Romaniuk, Ed., Wilga 2003. SPIE 5484; Photonics Applications in Astronomy, Communications, Industry, and High-Energy Physics Experiments 2003, 2004, 734 pages, 94 papers.

[3] —- Wilga 2004. SPIE 5775; Photonics Applications in Astronomy, Communications, Industry, and High-Energy Physics Experiments 2005, 2005, 710 pages, 92 papers.

[4] R. S. Romaniuk, S. Simrock, and V. M. Lutkovski, Eds., Wilga 2005 bis. SPIE 5948, Photonics Applications in Industry and Research 2005, 2005, 864 pages, 89 papers.

[5] R. S. Romaniuk, Ed., Wilga 2005. SPIE 6159; Photonics Applications in Astronomy, Communications, Industry, and High-Energy Physics Experiments 2005, 2006, 1244 pages, 172 papers.

[6] - Wilga 2006. SPIE 6347; Photonics Applications in Astronomy, Communications, Industry, and High-Energy Physics Experiments 2006 , 2006, 874 pages, 111 papers.

[7] — Wilga 2007. SPIE 6937; Photonics Applications in Astronomy, Communications, Industry, and High-Energy Physics Experiments 2007. 2008, 1274 pages, 152 papers.

[8] R. S. Romaniuk and T. R. Woliński, Eds., Wilga 2008. SPIE 7124; Photonics Applications in Astronomy, Communications, Industry, and High-Energy Physics Experiments 2008, 2008, 312 pages, 35 papers.

[9] R. S. Romaniuk and K. S. Kulpa, Eds., Wilga 2009. SPIE 7502; Photonics Applications in Astronomy, Communications, Industry, and High-Energy Physics Experiments 2009, 2009, 786 pages, 100 papers.

[10] R. S. Romaniuk, Ed., Wilga 2010. SPIE 7745; Photonics Applications in Astronomy, Communications, Industry, and High-Energy Physics Experiments 2010, 2010, 650 pages, 73 papers.

[11] — Wilga 2011. SPIE 8008; Photonics Applications in Astronomy, Communications, Industry, and High-Energy Physics Experiments 2011 , 2011, 500 pages, 70 papers.

[12] — Wilga 2012. SPIE 8454; Photonics Applications in Astronomy, Communication, Industry,andHighEnergy Physics Experiments 2012, 2012, 600 pages, 90 papers.

[13] J. Dorosz and R. Romaniuk, "The role of regional developments in optical fiber technology and photonics," Proceedings of SPIE, vol. 5028, pp. xi-xii, 2003.

[14] R. Romaniuk and K. Pozniak, "Foreword: Photonics and electronics for astronomy and high energy physics experiments in Poland," Proceedings of SPIE, vol. 5125, pp. xiii-xxxiv, 2002.

[15] W. Woliński, Z. Jankiewicz, and R. Romaniuk, "Proceedings of SPIE - The International Society for Optical Engineering: Introduction," Proceedings of SPIE, vol. 5230, pp. ix-x, 2003.

[16] R. Romaniuk, "Proceedings of SPIE - The International Society for Optical Engineering: Introduction," Proceedings of SPIE, vol. 5775, pp. xxi-xxvii, 2005.

[17] — , "Proceedings of SPIE - The International Society for Optical Engineering: Introduction," Proceedings of SPIE, vol. 5848, pp. xviixxi, 2005 .

[18] — , "Proceedings of SPIE - The International Society for Optical Engineering: Introduction," Proceedings of SPIE, vol. 6347, pp. xxixxxxii, 2006.

[19] W. Wolinski, Z. Jankiewicz, and R. Romaniuk, "Proceedings of SPIE - The International Society for Optical Engineering: Introduction," Proceedings of SPIE, vol. 6598, pp. ix-xii, 2007.

[20] R. Romaniuk, "Proceedings of SPIE - The International Society for Optical Engineering: Introduction,” Proceedings of SPIE, vol. 6937, pp. xxix-xli, 2008

[21] W. Wolinski, Z. Jankiewicz, and R. Romaniuk, "Proceedings of SPIE - The International Society for Optical Engineering: Introduction," Proceedings of SPIE, vol. 5229, pp. xi-xii, 2003.

[22] J. Dorosz, R. Romaniuk, and T. Wolinski, "Eleventh conference on optical fibers and their applications," Proceedings of SPIE, vol. 7120, pp. xiii-xv, 2008.

[23] R. Romaniuk and K. Kulpa, "Photonics applications in Astronomy, Communications Industry and High-Energy Physics Experiments 2009: Introduction," Proceedings of SPIE, vol. 7502, pp. xxiii-xxiv, 2009, art. no. 750201.

[24] R. Romaniuk, "Photonics and Web Engineering in Poland, WILGA 2009," Proceedings of SPIE, vol. 7502, 2009, art. no. 750202.

[25] — , "WILGA Symposium on photonics applications," Photonics Letters of Poland, vol. 1, no. 2, pp. 46-48, 2009. 
[26] — "Wilga 2010, Photonics Applications," Proceedings of SPIE, vol. 7745, pp. xiii-xviii, 2010

[27] _ "Wilga 2011, Photonics Applications," Proceedings of SPIE, vol. 8008, pp. xii-xviii, 2011.

[28] — "Wilga 2012, Photonics Applications," Proceedings of SPIE, vol. 8454, pp. vii-x, 2012

[29] _ - "Wilga photonics and web engineering 2010," Photonics Letters of Poland, vol. 2, no. 2, pp. 55-57, 2010.

[30] _ "Petabit photonic internet," Photonics Letters of Poland, vol. 3, no. 2, pp. 91-93, 2011.

[31] _ , "the Photonics Letter of Poland' A new peer-reviewed internet publication of the Photonics Society of Poland," Photonics Letters of Poland, vol. 1, no. 1, pp. 1-3, 2009.

[32] - "Wilga symposium on photonics applications," Photonics Letters of Poland, vol. 1, no. 2, pp. 46-48, 2009.

[33] — "POLFEL - A free electron laser in Poland," Photonics Letters of Poland, vol. 1, no. 3, pp. 103-105, 2009.

[34] R. S. Romaniuk, "Polish Debut," Photonics Spectra, vol. 26, no. 5, pp. 10-12, May 1992.
[35] — "The photonics scene in the new Poland," Photonics Spectra, vol. 26, no. 4, pp. 64-65, April 1992.

[36] J. Modelski and R. Romaniuk, "Electronics and telecommunications in Poland, issues and perspectives, Part I: Society and education," Proceedings of SPIE, vol. 7745, 2010, art. no. 774504.

[37] _ "Electronics and telecommunications in Poland, issues and perspectives, Part II: Science, research, development, higher education," Proceedings of SPIE, vol. 7745, 2010, art. no. 774505.

[38] _ "Electronics and telecommunications in Poland, issues and perspectives, Part III: Innovativeness, applications, economy, development scenarios, politics," Proceedings of SPIE, vol. 7745, 2010, art. no. 774506 .

[39] S. Chatrchyan, G. Asova, K. Pozniak, R. Romaniuk, and W. Zabolotny et al., "The CMS Collaboration, Observation of a new boson at a mass of $125 \mathrm{GeV}$ with the CMS experiment at the LHC," Physics Letters B, vol. 716, no. 1 , pp. 30-61, 2012. 